\title{
The Growth of Hospice Care in U.S. Nursing Homes
}

\author{
Susan C. Miller, $\mathbf{P h D}^{1}$, Julie Lima, $\mathbf{P h D}^{2}$, Pedro L. Gozalo, $\mathbf{P h D}^{4,2}$, and Vincent Mor, $\mathbf{P h D}^{1,2}$ \\ ${ }^{1}$ Associate Professor of Community Health (Research), Center for Gerontology and Healthcare \\ Research and Department of Community Health, Alpert Medical School, Brown University, \\ Providence, RI \\ ${ }^{2}$ Senior Research Analyst, Center for Gerontology and Healthcare Research, Alpert Medical \\ School, Brown University, Providence, RI \\ ${ }^{3}$ Assistant Professor of Community Health (Research), Center for Gerontology and Healthcare \\ Research and Department of Community Health, Alpert Medical School, Brown University, \\ Providence, $\mathrm{RI}$ \\ ${ }^{4}$ Professor and Chair, Department of Community Health and Center for Gerontology and \\ Healthcare Research, Alpert Medical School, Brown University, Providence, RI
}

\section{Abstract}

Objectives-To inform efforts aimed at reducing Medicare hospice expenditures by describing the longitudinal use of hospice care in nursing homes (NHs) and examining how hospice provider growth is associated with use.

Design—Longitudinal study using NH resident assessment (MDS) and Medicare denominator and claims data for years 1999 through 2006.

Setting -Nursing homes in the 50 U.S. states and District of Columbia

Participants-Persons dying in U.S. NHs

Measurements-We identified Medicare beneficiaries dying in NHs, receipt of NH hospice, and lengths of hospice stay. We also identified the number of hospices providing care in NHs and used a panel data fixed-effect (within) regression analysis to examine how growth in providers affected hospice use.

Results-Between 1999 and 2006 the number of hospices providing care in NHs rose from 1,850 to 2,768 and rates of $\mathrm{NH}$ hospice use more than doubled (14\% to $33 \%$ respectively). With this growth came a doubling of mean lengths of stay (from 46 to 93 days) and a 10\% increase in the proportion of NH hospice decedents with noncancer diagnoses (69\% in 1999 to $83 \%$ in 2006). Controlling for time trends, for every ten new hospice providers within a state there was an average state increase of $0.58 \%$ (95\% confidence interval: $0.383,0.782$ ) in NH hospice use. Much state variation in $\mathrm{NH}$ hospice use and growth was observed.

${ }^{1}$ Corresponding Author: 121 South Main Street, Providence, RI 02889; (401) 863-9216 (401) 863-9219 (fax); Susan_Miller@brown.edu.

2 Alternate Corresponding Author: 1316 Old Oak Trail, Flower Mound, TX 75028; Phone: (972) 355-7814; Julie_Lima@brown.edu Conflict of Interest: Miller, Gozalo and Mor are funded by the National Hospice Foundation to design and simulate modifications to the Medicare hospice benefit payment system

Author Contributions: Miller contributed to the concept and drafting of the manuscript and Miller and Mor contributed to the acquisition of data. All authors contributed to the design, analysis and interpretation of data, and to the critical review of the manuscript.

Sponsor's Role: None 
Conclusion-Policy efforts to curb Medicare hospice expenditures (driven in part by provider growth) must consider the potentially negative impact of changes on access for dying (mostly noncancer) $\mathrm{NH}$ residents.

\section{Keywords}

hospice; nursing homes; end of life; Medicare; reimbursement policy

\section{Introduction}

A half million older adults die in U.S. nursing homes (NHs) each year. ${ }^{1}$ However, while NHs are increasingly sites of end-of-life care, ${ }^{1}$ there are concerns about the quality of that care. Pain assessment and management are often inadequate, ${ }^{2-8}$ and family members of dying residents report stress due to limited physician visits and insufficient staffing. ${ }^{9}$ The use of Medicare hospice care to augment end-of-life care in $\mathrm{NHs}$ is a viable option for addressing such concerns and there is a long history of NH-hospice collaborations; ${ }^{10}$ in fact, in $200478 \%$ of U.S. NHs contracted with hospice providers. ${ }^{11}$ Documented benefits of NH hospice care provided to $\mathrm{NH}$ residents include better pain management, ${ }^{12}$ fewer hospitalizations, ${ }^{13}$ greater family satisfaction with end-of-life care, ${ }^{14-16}$ and lower costs (at least in the last month of life) ${ }^{17}{ }^{18}$ However, the increasingly long lengths of hospice stay and their higher prevalence in $\mathrm{NHs}^{19}$ has resulted in closer scrutiny of $\mathrm{NH}$ hospice care ${ }^{20}$ and prompted consideration of policies that might jeopardize access. Also, some have advocated for NH hospice's dissolution with an argument in part based on the premise of underuse. ${ }^{21,22}$

Concerns regarding increased Medicare hospice expenditures prevail and expenditure increases are believed in part to have been driven by the large growth in new Medicarecertified hospice providers. ${ }^{19,23}$ Between 2000 and 2007 there was a five percent average annual growth of Medicare hospice providers and this growth was almost entirely attributable to growth among proprietary providers. This growth has been accompanied by a ten percent average annual growth in Medicare hospice beneficiaries and large increases in lengths of hospice stay. ${ }^{19}, 23$ Consequently, Medicare hospice expenditures have escalated with long lengths of hospice stays contributing substantially to this increase. Longer stays are believed to be driven in part by financially motivated provider behavior since, regardless of length of stay, hospices are paid the same daily rates for routine hospice home care $(95 \%$ of all hospice care days) and a hospice's profit margin is greater when its stays are longer. ${ }^{19}$ Therefore, the Medicare Payment Advisory Commission (MedPAC) has speculated that some hospices selectively admit patients with higher probabilities of long stays, and these persons are more often persons with noncancer diagnoses. ${ }^{19}$ Given the high prevalence of noncancer diagnoses in NHs, the concern is that policy efforts to decrease long hospice stays may differentially affect access in NHs, even though hospice has been found to be beneficial and potentially cost-saving. ${ }^{2}, 4,9,12-18$

Both excessively short and long NH hospice stays are problematic (from a expenditure perspective); very short stays lose opportunities for Medicare savings by reducing end-oflife hospitalizations and very long stays increase overall Medicare costs. ${ }^{18,}{ }^{19}$ The challenge in NHs is how to balance the need for hospice access with the timing of referral for NH residents in the last stages of (noncancer) chronic terminal illnesses for which predicted survival is poor, at best. ${ }^{24-26}$ From a policy perspective, the challenge is how to curb behavior by some providers who may be exploiting a payment system that results in higher profits for longer hospice stays ${ }^{19}$ without jeopardizing access in the NH setting altogether. 
Missing from a recent MedPAC report ${ }^{19}$ and a related commentary ${ }^{23}$ on growth of Medicare hospice providers and hospice use is discussion and evidence documenting the longitudinal use of hospice by the half million older adults dying in NHs each year. ${ }^{1}$ Unknown is how growth in hospice providers and in hospice use and lengths of stay are different for care provided in NHs. The population-based longitudinal study presented here provides this information. Using national data from 1999 through 2006 this study describes growth in the rates of hospice use and in mean lengths of stay and it examines the effect of $\mathrm{NH}$ provider growth on rates of hospice use. Furthermore, this study presents data on the proportion of hospice care provided in NHs between 1999 and 2006 and contrasts the differing use of NH hospice across U.S. states.

\section{Methods}

\section{Data and Study Population}

For years 1999 through 2006 we used NH resident assessment (MDS) data for the 50 U.S. states and District of Columbia, Medicare Part A claims data (i.e., for hospice, hospital, home health, outpatient and skilled nursing facility [SNF] care), and Medicare enrollment data (which includes vital statistics data) to create a patient history for all residents. ${ }^{27} \mathrm{We}$ concatenated MDS and claims records to create a per resident history file to determine where study subjects were located and the care they received in the days and weeks prior to death.

The population of NH residents was first identified by using 1999-2006 MDS data from Medicare/Medicaid certified NHs (97\% of US NHs). Then, to determine Medicare eligibility and if and when death occurred, these data were matched to the Medicare denominator files. Across study years residents' MDS/denominator match rate was over 90 percent. For 2006, there was a 93.3\% match rate resulting in the identification of 3,090,244 $\mathrm{NH}$ residents in 2006. Also, to determine the proportion of Medicare beneficiaries who received hospice in NHs (see below) we retrieved data on the total number of Medicare hospice beneficiaries in a calendar year from Medicare and Medicaid Statistical Reports (2001-2008). ${ }^{28}$

\section{Nursing Home Decedents and NH Hospice}

Nursing home decedents were defined as Medicare beneficiaries whose deaths occurred within one day of an identified NH stay or within seven days of hospice transfer from a NH (as done in previous research). ${ }^{13,}{ }^{29}$ In study year 1999, to enable capture of at least 180 days of a hospice stay, we only included NH residents who died in July through December. Nursing home hospice use was identified when dates on hospice claims overlapped with dates of NH stays, and decedents classified as receiving NH hospice did not necessarily die with hospice. For instance, in 2006 nine percent of $\mathrm{NH}$ decedents receiving hospice were discharged from hospice prior to death. The rates of NH hospice use (for the U.S. and individual states and DC) were determined by dividing the number of $\mathrm{NH}$ decedents in a calendar year receiving $\mathrm{NH}$ hospice care by the total number of $\mathrm{NH}$ decedents in that calendar year.

Decedent Characteristics-Data from the MDS prior to death were used to determine a resident's gender, race/ethnicity and age. Race was categorized as White (non-Hispanic) and Minority (including Hispanic). Age was categorized as less than 65, 65 to 84 and 85+. The principal diagnoses on hospice claims were used to determine hospice residents' diagnoses, and diagnoses were categorized as being noncancer or cancer (primary and secondary cancer codes as well as codes for lymphatic and hematopoietic neoplasms [from International Classifications of Diseases $9^{\text {th }}$ Edition, Clinical Modification]). To determine the proportion of cancer and noncancer $\mathrm{NH}$ decedents accessing hospice we used the documentation of a 
cancer diagnosis on the MDS as evidence of a cancer diagnosis since the denominator included both residents with and without hospice claims.

Days of Hospice Care-For NH decedents receiving NH hospice care, dates on hospice claims were used to identify the hospice length of stay (i.e., total number of days on the Medicare hospice benefit). When more than one hospice episode occurred (i.e., individual was discharged and readmitted to hospice), the days from each episode were totaled. Hospice days prior to $\mathrm{NH}$ admission were included in totals when these days were part of a continuous hospice episode or when the episode(s) occurred within six months of the $\mathrm{NH}$ hospice episode. For 2006, 12,950 (7.6\%) of the 172,015 NH hospice decedents received hospice care prior to NH admission, and of these, 10,763 (83\%) had one continuous hospice episode.

Hospices Providing Care in NHs-Using the hospice claims for NH residents, we identified the unique number of hospices providing care in NHs in 1999 through 2006. The provider's official address in the hospice provider of service file (or if unavailable, the first two digits of the provider number) were used to assign the hospice to a state. Through previous analysis we had determined the growth of providers in NHs to be different than the growth of all Medicare certified hospice providers. Specifically, using 1999 as the base year, growth in the number of hospices providing care in NHs began in 2000 and continued through 2006 (see Figure 1). However, growth in the number of all Medicare certified hospice providers did not occur until 2003. ${ }^{19}$

Proportion of Medicare Hospice Care in NHs-This proportion was defined as the percentage of total Medicare hospice beneficiaries in a calendar year who received any hospice while in a NH. The numerator was the total number of $\mathrm{NH}$ residents in a calendar year with any $\mathrm{NH}$ hospice (not restricted to decedents) and the denominator was the total number of Medicare beneficiaries in a calendar year who received hospice (from the Medicare and Medicaid Statistical Reports). ${ }^{28}$

\section{Analytic Approach}

Descriptive statistics were used to show provider growth, hospice decedent characteristics, and the rate of NH hospice use (weighted by number of deaths in individual states and U.S.) over time and in 2006. T-test and chi square statistics were used to test the statistical significance of observed differences, and a Breslow-Day statistic was used to test for interactions (i.e., for changes between 1999 and 2006 by categories of U.S. states). To examine how the growth in hospice providers affected NH hospice use, we performed a panel data fixed-effect (within) regression analysis that controlled for differences across states (50 U.S. states and District of Columbia). This analysis included a year indicator to capture the secular trend (scaled to be zero in the first year [1999]) and the number of hospices (scaled to be zero in 1999 and representing an increase of ten providers per unit of change). State-level observations were weighted in the regression by their number of confirmed NH decedents to obtain nationally representative estimates.

\section{Results}

While the number of Medicare hospice beneficiaries in NHs doubled from 101,843 in 1999 to 233,844 in 2006 , the proportion of beneficiaries who were in NHs largely mirrored the overall growth in the Medicare hospice program. In 1999, 21.7 percent of Medicare hospice beneficiaries resided in NHs; this rose to $24.2 \%$ in 2002 and remained constant through 2006, at approximately 25 percent (data not shown). 
The number of Medicare-certified hospices providing care in US NHs was 1,850 in 1999 and 2,768 in 2006 (a 49.6\% growth), and the rate of $\mathrm{NH}$ decedent hospice use was $14.0 \%$ in 1999 and $33.1 \%$ in 2006 (a growth of 137\%; Figure 1). Using regression analysis that controlled for state differences and secular time trend, we found that for every ten new hospice providers within a state there was an average state increase of $0.58 \%$ in $\mathrm{NH}$ hospice use (95\% confidence interval: 0.383, 0.782). Between 1999 through 2006 this effect represents, on average, an additional four percent increase in hospice use in the ten states with the largest provider growth, compared to the ten states with the lowest growth. Of note, NH hospice use increased significantly over time irrespective of provider growth.

Controlling for hospice provider growth and differences across states, rates of hospice use grew each year by an average of $2.5 \%$ (AOR $2.50 ; 95 \%$ CI 2.375, 2.620).

The demographic characteristics of NH hospice decedents changed little across study years. Over time, most hospice decedents were female (about 67\%), White (around 90\%), and 50 to $55 \%$ were 85 years of age or older. However, the proportion of $\mathrm{NH}$ hospice decedents with noncancer diagnoses grew from 69.2\% in 1999 to $82.6 \%$ in 2006 (p <.001) and there was significant growth in the proportion of cancer and noncancer decedents accessing hospice. In 1999, $11.9 \%$ of $\mathrm{NH}$ decedents with noncancer diagnosis and $23.0 \%$ with cancer diagnoses accessed hospice; in 2006, these rates rose to $31.4 \%$ and $51.3 \%$ respectively ( $\mathrm{p}<$. 001).

With the growth in NH hospice use came longer mean lengths of hospice stay (Figure 2). Nationwide, mean stays for NH decedents rose from 45.8 days in 1999 to 93.2 days in 2006 ( $\mathrm{p}<.001$ ), and this increase appears to have been driven by higher proportions of long hospice stays. For instance, the proportion of hospice stays over 180 days rose from $6.6 \%$ in 1999 to $15.6 \%$ in 2006 ( $\mathrm{p}<.001$ ) while the proportion of hospice stays of seven days or less remained relatively stable at around $30 \%$.

Table 1 presents the considerable state variation in the growth of $\mathrm{NH}$ hospice providers and in NH hospice use between 1999 and 2006. In 2006, the lowest rates of NH hospice use were in Alaska (2.3\%) and Hawaii (7.9\%), but rates were also low in several rural states such as Wyoming (9.0\%) and Vermont (8.1\%). Even in these low use states, however, the percent of growth in NH hospice use was high; for example, in Wyoming the increase in the rate of hospice use was 163 percent and in Vermont it was 96 percent. In the ten states with the most provider growth (Alabama, Georgia, Idaho, Louisiana, Mississippi, New Mexico, Oklahoma, South Carolina, Tennessee and Utah; see Table 1), there was a (weighted) 192\% increase in rates of NH hospice use between 1999 and 2006 compared to a (weighted) 101\% increase in the ten states with the least provider growth (Arkansas, Florida, Kentucky, Maryland, Minnesota, New Hampshire, New York, North Dakota, Minnesota, South Dakota and Washington; see Table 1). However, the proportion of Medicare hospice care in NHs differed little in 2006 for these states with the most versus least provider growth (21.6 versus 22.6 respectively).

Mean lengths of stay also differed in the ten states with the most versus the least provider growth, with mean stays being significantly longer in states with the most versus least growth (131 versus 95 days; $p<.001$ ). Additionally, increases in lengths of stay at the $90^{\text {th }}$ percentile were greater in the ten states with the most versus the least provider growth. At the $90^{\text {th }}$ percentile, hospice stays were 173 days in 1999 and 399 days in 2006 (a 131\% increase) in states with the most provider growth while they were 141 and 276 days (respectively; a 96\% increase) in states with the least growth. However, increases in the proportion of $\mathrm{NH}$ hospice decedents with noncancer diagnoses were similar for the ten states with the most versus the least provider growth (13.8\% versus $13.0 \%$ increase). 


\section{Discussion}

There was substantial growth in the number of hospices providing care in NHs and this growth was significantly associated with increased rates of hospice use. Furthermore, increased rates of hospice use were accompanied by dramatic increases in mean hospice stays. Herein lays the concern. While increased access to hospice care in NHs had been promoted ${ }^{30}$ increased access appears to come at a cost of longer stays and these have resulted in increased Medicare hospice expenditures. ${ }^{19}$ Furthermore, although increases in hospice use are a likely response to unmet needs, ${ }^{24,} 25$ greater growth in long stays were observed in the ten states with the most versus the least provider growth even though both groups had similar increases in noncancer patients. Further study is needed to understand the provider and/or healthcare market factors influencing these differences.

In the US, $67 \%$ of older persons with dementia-related diagnoses and $28 \%$ of persons with other noncancer diagnoses die in NHs, while only $21 \%$ of those with cancer diagnoses die in NHs. ${ }^{1}$ Therefore, to provide hospice care to all but a very small proportion of dying $\mathrm{NH}$ residents means substantial care will be provided to persons with noncancer diagnoses, a high proportion of who will have dementia-related diagnoses. We observed this firsthand since the proportion of hospice $\mathrm{NH}$ decedents with noncancer diagnoses was sizable (at $69 \%$ ) even prior to the substantial growth of hospice providers, when only $14 \%$ of decedents accessed hospice. However, while $83 \%$ of $\mathrm{NH}$ decedents had noncancer diagnoses in 2006, only $31 \%$ percent accessed hospice. In contrast, $51 \%$ of $\mathrm{NH}$ decedents with cancer diagnoses accessed hospice in 2006. This large discrepancy in access reflects the continuing barriers to Medicare hospice for persons with (noncancer) chronic terminal illnesses in $\mathrm{NH}$ and non-NH settings. ${ }^{24-26}$

Mean hospice stays for NH decedents more than doubled, from 46 days in 1999 to 93 days in 2006. Additionally, in 2006 the 93 day mean hospice stay for NH decedents is estimated to be at least 20 days longer than the mean stay for non-NH hospice decedents (based on the proportion of all Medicare hospice provided in NHs, the mean NH stay and on MedPAC length of stay data for all hospice decedents). ${ }^{19}$ This longer mean stay for $\mathrm{NH}$ decedents is consistent with NH case-mix differences (as discussed above) and with the more bimodal distribution of hospice lengths of stay for persons with noncancer diagnosis. ${ }^{31}$ Still, as discussed above, it appears differing provider and/or healthcare market behavior may influence the presence of longer long stays (days of stay at $90^{\text {th }}$ percentile) since increases in these days were significantly greater in the ten states with the greatest versus least provider growth.

Our study provides new information showing almost a third of Medicare beneficiaries dying in NHs accessed Medicare hospice in 2006, and given the observed trends this growth in use is likely continuing. Additionally, other recent research has shown $40 \%$ of $\mathrm{NH}$ decedents with end-stage dementia and 35\% of those with a co-morbid dementia diagnoses accessed hospice in 2006. ${ }^{32}$ Therefore, calls for elimination of Medicare hospice in NHs based on its underuse appear unfounded, ${ }^{21,} 22$ especially considering our finding that use in the $\mathrm{NH}$ mirrored the overall growth in Medicare hospice use. Additionally, the notion that the complexity of NH-hospice collaborations is a major obstacle to the viability of hospice care in $\mathrm{NHs}^{22}$ also appears unfounded given the high proportion of U.S. NHs contracting with hospice providers and the documented outcomes resulting from this collaborative care. ${ }^{4,9}$, ${ }^{12-16}$ Nevertheless, the argument that the design of the Medicare hospice benefit creates barriers to its access ${ }^{21,22}$ is undeniable.

There are major shortcomings in the Medicare hospice benefit that appear to influence the timing of referral (resulting in very short and long stays), ${ }^{33}$ and that may limit further 
increases in access for NH residents. First, the need for a physician-certified six-month terminal prognosis creates substantial barriers for persons with (noncancer) chronic terminal illnesses for whom a six-month prognosis is difficult. ${ }^{24,}{ }^{25}$ While this barrier affects persons dying in NHs and in other settings (and 72\% of persons with non-dementia chronic terminal illnesses die in non-NH settings), ${ }_{1}^{1}$ the barrier is particularly important in $\mathrm{NHs}$ given the high proportion of $\mathrm{NH}$ residents with chronic terminal illnesses. Another major barrier arising from the Medicare hospice benefit is the requirement that beneficiaries enrolling in hospice must forgo other Medicare-Part A care (when such care is related to the terminal illness). For beneficiaries in the community or in NHs this means hospital care and curative treatment must be abandoned, but it often also means abandonment of expensive treatments such as blood transfusions or palliative radiation (when hospices lack financial resources to support such care). ${ }^{24-26}$ Additionally, in NHs the Medicare Part A forfeiture requirement creates a system-wide barrier since dying NH residents routinely receive Medicare Part-A skilled nursing facility (SNF) care (after hospitalizations), ${ }^{34}$ and its forfeiture is financially disadvantageous for NHs and families. ${ }^{35}$ Even given these barriers, we did find a third of $\mathrm{NH}$ decedents in 2006 enrolled in Medicare hospice. However, as expected, a high proportion of hospice stays were seven days or less (30.6\% in 2006) or over 181 days (15.6\% in 2006; Table 2).

While most U.S. NHs approach the provision of end-of-life palliative care by referring residents to hospice, many NHs also invest in developing internal palliative care programs/ expertise (with or without hospice care). ${ }^{11}$ The few studies that have tested the integration of (nonhospice) palliative care expertise and processes into NHs have shown improvement in care processes, ${ }^{36-40}$ and when combined with a quality improvement program, a reduction in pain prevalence. ${ }^{41}$ However, there has been no widespread dissemination of any of these efforts. In 2004, $27 \%$ of U.S. nursing homes reported having special programs and (specially) trained staff for hospice or palliative/end-of-life care but information on the scope of practices included in these programs was not reported. ${ }^{11}$ More study is needed to understand the extent and breadth of nonhospice palliative care provision and expertise in U.S. NHs, the resulting quality outcomes, and the feasibility of its widespread implementation. Without a more in-depth understanding of what $\mathrm{NH}$ practices and investments result in higher quality palliative care outcomes and whether the few smallscale studies can be replicated across a broad range of NHs, paying NHs more to provide "palliative care" in lieu of paying Medicare-certified hospices ${ }^{21}$ is ill-advised. At present, given our current understanding of NH end-of-life care and of the benefits and use of hospice care in NHs, ${ }^{5-8}, 14,16$ the provision of NH-hospice collaborative care appears to be the most feasible option for widespread improvement of dying residents' quality of care and life. ${ }^{10}$ Notwithstanding this, increased palliative care knowledge and (availability to) expertise in NHs and in other healthcare settings continues to be needed to ensure highquality symptom management to persons who do not qualify for or choose hospice, and payment or quality indicator oversight efforts to incentivize such symptom management are desirable.

The MedPAC has recommended changing the Medicare payment system so the per diem rate for hospice routine home care (95\% of all hospice care days) better reflects the intensity of hospice service provision. ${ }^{19}$ Since research has shown hospice visits to be more frequent at time periods closer to the beginning and end of hospice episodes, ${ }^{42-44}$ it is reasonable to have higher payment rates around the time of hospice admission and around the time a patient is dying. The MedPAC has also recommended closer scrutiny of recertification of hospice patients after the $180^{\text {th }}$ day of stay, specifically, they propose requiring patient visits by physicians or advanced practice nurses to evaluate their continued eligibility and need for care. ${ }^{19}$ Together, these recommended approaches to curbing Medicare costs appear to be a viable option for both retaining access while reducing the number of long (costly) hospice 
stays (some of which appear to result from perverse financial incentives). However, evaluation of such a payment option must include consideration of variations in hospice use in NHs (as shown here) and the probable greater impact of changes on hospice access for dying $\mathrm{NH}$ residents, given the high proportion of persons with noncancer diagnoses in NHs.

In conclusion, given the increasingly high proportion of Medicare beneficiaries who die in NHs and their entitlement to and benefit from the Medicare hospice benefit, denying them access to NH hospice is not desirable. Our analyses provide new information on the increased use of hospice care in NHs and show substantial increases in very long hospice stays. As such, our data support the need for a modification in the current Medicare hospice reimbursement systems which would vary payments as a function of length of stay. ${ }^{19}$ However, it is important for any new policy to explicitly acknowledge the challenges inherent in the timing of hospice referral for $\mathrm{NH}$ residents in the last stages of (noncancer) chronic terminal illnesses ${ }^{24-26}$ by recognizing "early" referrals will occur and deeming them "acceptable" in the presence of well-documented physician evaluations and eligibility determinations. Without such explicit acknowledgement the fear is that undue scrutiny may occur, resulting in decreasing enrollments and a higher prevalence of very short stays.

\section{Acknowledgments}

We wish to thank Mr. Venkatesh Nathilvar, MS who merged the datasets and assembled the study's analytic database.

This study was supported by a grant from the Agency for Health Care Research and Quality (R03HS016918) and by the Shaping Long Term Care in American Project funded by the National Institute on Aging (P01AG027296).

This paper was presented at the 2010 Annual Meeting of the American Association of Hospice and Palliative Medicine.

\section{References}

1. Mitchell SL, Teno JM, Miller SC, Mor V. A national study of the location of death for older persons with dementia. J Am Geriatr Soc. 2005; 53:299-305. [PubMed: 15673356]

2. Teno JM, Clarridge BR, Casey V, et al. Family perspectives on end-of-life care at the last place of care. JAMA. 2004; 291:88-93. [PubMed: 14709580]

3. Cohen-Mansfield J, Lipson S. Pain in cognitively impaired nursing home residents: How well are physicians diagnosing it? J Am Geriatr Soc. 2002; 50:1039-1044. [PubMed: 12110063]

4. Miller SC, Mor V, Teno J. Hospice enrollment and pain assessment and management in nursing homes. J Pain Symptom Manage. 2003; 26:791-799. [PubMed: 12967728]

5. Hanson LC, Eckert KJ, Dobbs D, et al. Symptom experience of dying long-term care residents. J Am Geriatr Soc. 2008; 56:91-98. [PubMed: 17727647]

6. Mitchell SL, Teno JM, Kiely DK, et al. The clinical course of advanced dementia. N Engl J Med. 2009; 361:1529-1538. [PubMed: 19828530]

7. Bernabei R, Gambassi G, Lapane K, et al. Management of pain in elderly patients with cancer. SAGE Study Group. Systematic Assessment of Geriatric Drug Use via Epidemiology. JAMA. 1998; 279:1877-1882. [PubMed: 9634258]

8. Ferrell BR, Dean GE, Grant M, Coluzzi P. An institutional commitment to pain management. J Clin Oncol. 1995; 13:2158-2165. [PubMed: 7666074]

9. Shield R, Wetle T, Teno J, et al. Physicians "missing in action": Family perspectives on physician and staffing problems in end-of-life care in the nursing home. J Am Geriatr Soc. 2005; 53:16511657. [PubMed: 16181162]

10. Miller SC. A model for successful nursing home-hospice partnerships. J Palliat Med. 2010 Apr 9. Epub ahead of print. 
11. Miller SC, Han B. End-of-life care in U.S. nursing homes: Nursing homes with special programs and trained staff for hospice or palliative/end-of-life care. J Palliat Med. 2008; 11:866-877. [PubMed: 18715179]

12. Miller SC, Mor V, Wu N, et al. Does receipt of hospice care in nursing homes improve the management of pain at the end of life? J Am Geriatr Soc. 2002; 50:507-515. [PubMed: 11943048]

13. Gozalo P, Miller S. Hospice enrollment and evaluation of its causal effect on hospitalization of dying nursing home patients. Health Serv Res. 2007; 42:587-610. [PubMed: 17362208]

14. Baer WM, Hanson LC. Families' perception of the added value of hospice in the nursing home. $\mathrm{J}$ Am Geriatr Soc. 2000; 48:879-882. [PubMed: 10968290]

15. Munn JC, Dobbs D, Meier A, et al. The end-of-life experience in long-term care: Five themes identified from focus groups with residents, family members, and staff. The Gerontologist. 2008; 48:485-494. [PubMed: 18728298]

16. Wetle, T.; Teno, J.; Shield, R.; Welch, LC.; Miller, SC. End of Life in Nursing Homes: Experiences and Policy Recommendations. Washington, D.C.: AARP Public Policy Institute; 2004.

17. Gozalo P, Miller S, Intrator O, et al. Hospice effect on government expenditures among nursing home residents. Health Serv Res. 2008; 43:134-153. [PubMed: 18211522]

18. Miller SC, Intrator O, Gozalo P, et al. Government expenditures at the end of life for short- and long-stay nursing home residents: Differences by hospice enrollment status. J Am Geriatr Soc. 2004; 52:1284-1292. [PubMed: 15271115]

19. Medicare Payment Advisory Commission. Report to the Congress: Medicare Payment Policy. Washington, DC: 2009. Reforming Medicare's Hospice Benefit; p. 347-376.

20. Office of Inspector General. Medicare Hospice Care for Beneficiaries in Nursing Facilities: Compliance with Medicare Coverage Requirements. 2009.

21. Huskamp HA, Stevenson DG, Chernew ME, et al. A new medicare end-of-life benefit for nursing home residents. Health Affairs. 2010; 29:130-135. [PubMed: 20048371]

22. Meier DE, Lim B, Carlson MD. Raising the standard: palliative care in nursing homes. Health Affairs. 2010; 29:136-140. [PubMed: 20048372]

23. Iglehart JK. A new era of for-profit hospice care--the Medicare benefit. N Engl J Med. 2009; 360:2701-2703. [PubMed: 19553645]

24. Wright A, Katz I. Letting go of the rope--aggressive treatment, hospice care, and open access. N Engl J Med. 2007; 357:324-327. [PubMed: 17652646]

25. Gazelle G. Understanding hospice--an underutilized option for life's final chapter. N Engl J Med. 2007; 357:321-324. [PubMed: 17652645]

26. Welch LC, Miller SC, Martin EW, et al. Referral and timing of referral to hospice care in nursing homes: The significant role of staff members. The Gerontologist. 2008; 48:477-484. [PubMed: 18728297]

27. Intrator O, Berg K, Hiris V, et al. Development and validation of the Medicare MDS Residential History File. Gerontologist. 2003; 43:30-31.

28. Centers for Medicare \& Medicaid Services. Medicare \& Medicaid Statistical Supplement. 2001-2008.

29. Miller S, Mor V, Wu N, et al. Does receipt of hospice care in nursing homes improve the management of pain at the end of life? J Am Geriatr Soc. 2002; 50:507-515. [PubMed: 11943048]

30. Zerzan J, Stearns S, Hanson L. Access to palliative care and hospice in nursing homes. JAMA. 2000; 284:2489-2494. [PubMed: 11074779]

31. Miller SC, Mor V. The emergence of Medicare hospice care in US nursing homes. PalliatMed. $2001 ; 15: 471-480$.

32. Miller, S.; Lima, J.; Mitchell, S., et al. Gerontologist. Trends in Hospice use among Nursing Home Decedents with Dementia: 1999 to 2006. Annual Meeting of Gerontological Society of America; 2009.

33. Miller SC, Weitzen S, Kinzbrunner B. Factors associated with the high prevalence of short hospice stays. JPalliatMed. 2003; 6:725-736. 
34. Levy CR, Fish R, Kramer AM. Site of death in the hospital versus nursing home of medicare skilled nursing facility residents admitted under Medicare's Part A benefit. J Am Geriatri Soc. 2004; 52:1247-1254.

35. Miller SC, Teno JM, Mor V. Hospice and palliative care in nursing homes. Clin Geriatr Med. 2004; 20:717-734. vii. [PubMed: 15541622]

36. Hanson LC, Reynolds KS, Henderson M, et al. A quality improvement intervention to increase palliative care in nursing homes. J Palliat Med. 2005; 8:576-584. [PubMed: 15992199]

37. Keay TJ, Alexander C, McNally K, et al. Nursing home physician educational intervention improves end-of-life outcomes. J Palliat Med. 2003; 6:205-213. [PubMed: 12854937]

38. Rochon, T.; Evans, B.; Dexter, C., et al. Initial development and evaluation of a nurse practitioner palliative care consultation service. Poster abstract \#75617. Las Vegas, NV. AGS Annual Scientific Meeting; 2004.

39. Tuch H, Parrish P, Romer AL. Integrating palliative care into nursing homes. J Palliat Med. 2003; 6:297-309. [PubMed: 12854950]

40. Weissman DE, Griffie J, Muchka S, et al. Improving pain management in long-term care facilities. J Palliat Med. 2001; 4:567-573. [PubMed: 11798492]

41. Baier RR, Gifford DR, Patry G, et al. Ameliorating pain in nursing homes: A collaborative qualityimprovement project. J Am Geriatr Soc. 2004; 52:1988-1995. [PubMed: 15571532]

42. Miller SC. Hospice care in nursing homes: Is site of care associated with visit volume? J Am Geriatr Soc. 2004; 52:1331-1336. [PubMed: 15271122]

43. Medicare Payment Advisory Commission. Report to Congress: Reforming the Delivery System. Washington, DC: 2008. Evaluating Medicare's Hospice Benefit; p. 203-240.

44. Gruneir A, Miller S. Hospice care in the nursing home: Changes in visit volume from enrollment to discharge among longer-stay residents. J Pain Symptom Manage. 2006; 32:478-487. [PubMed: 17085274] 
Figure 1. Percentage Growth in Hospices Providing Care in Nursing Homes and Rates of Nursing Home Decedent Hospice Use, 1999-2006

Bar $=$ Percent of Nursing Home Decedents Accessing Hospice

Line $=$ Percent Growth in Number of Hospice Providers Since 1999 


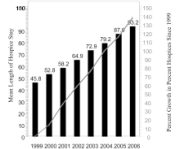

Figure 2. Percentage Growth in Rates of Hospice Use and Mean Lengths of Hospice Stay for Nursing Home Decedents, 1999-2006

Bar $=$ Mean Length of Hospice Stay

Line $=$ Percent Growth in Percent Hospice Since 1999 


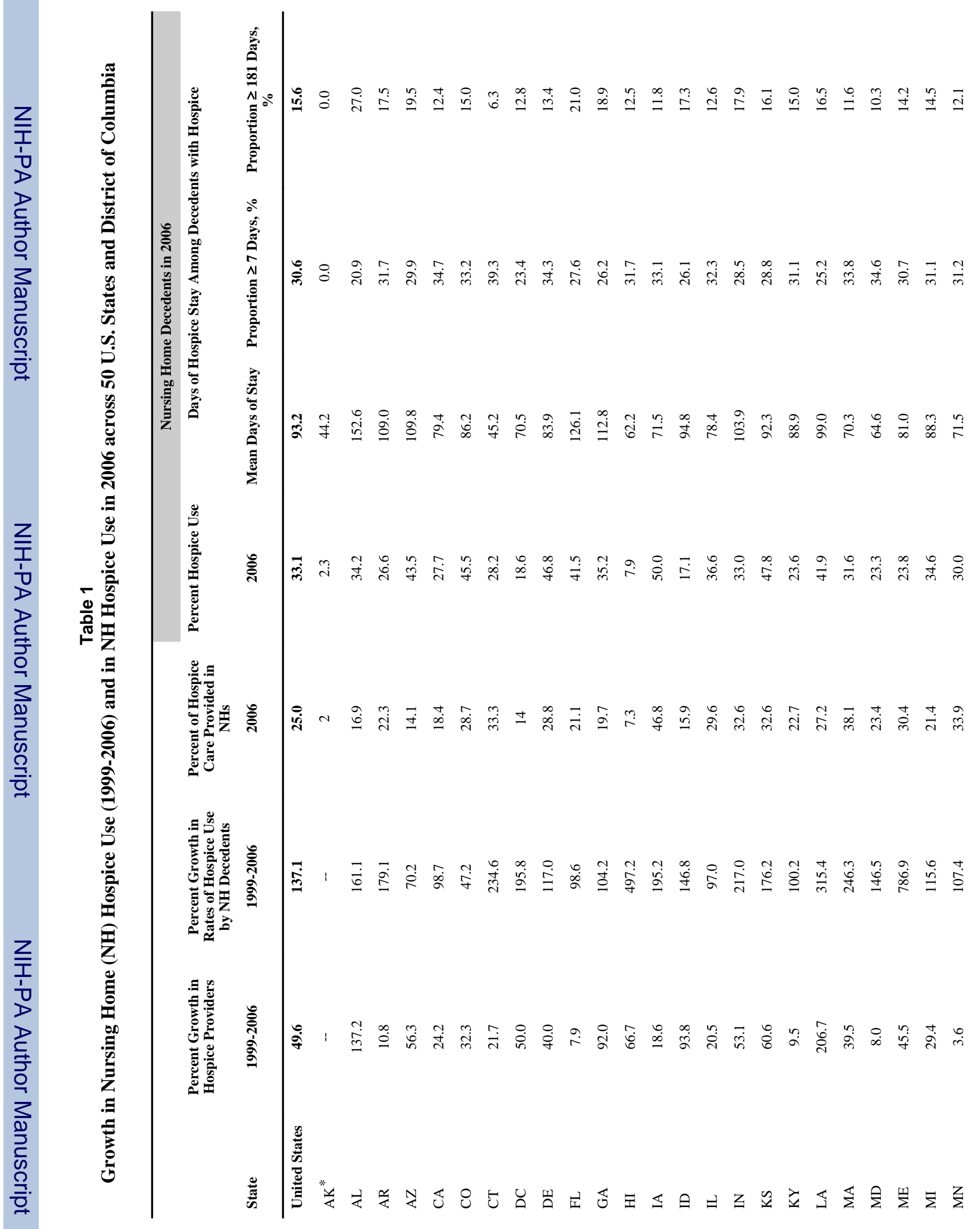




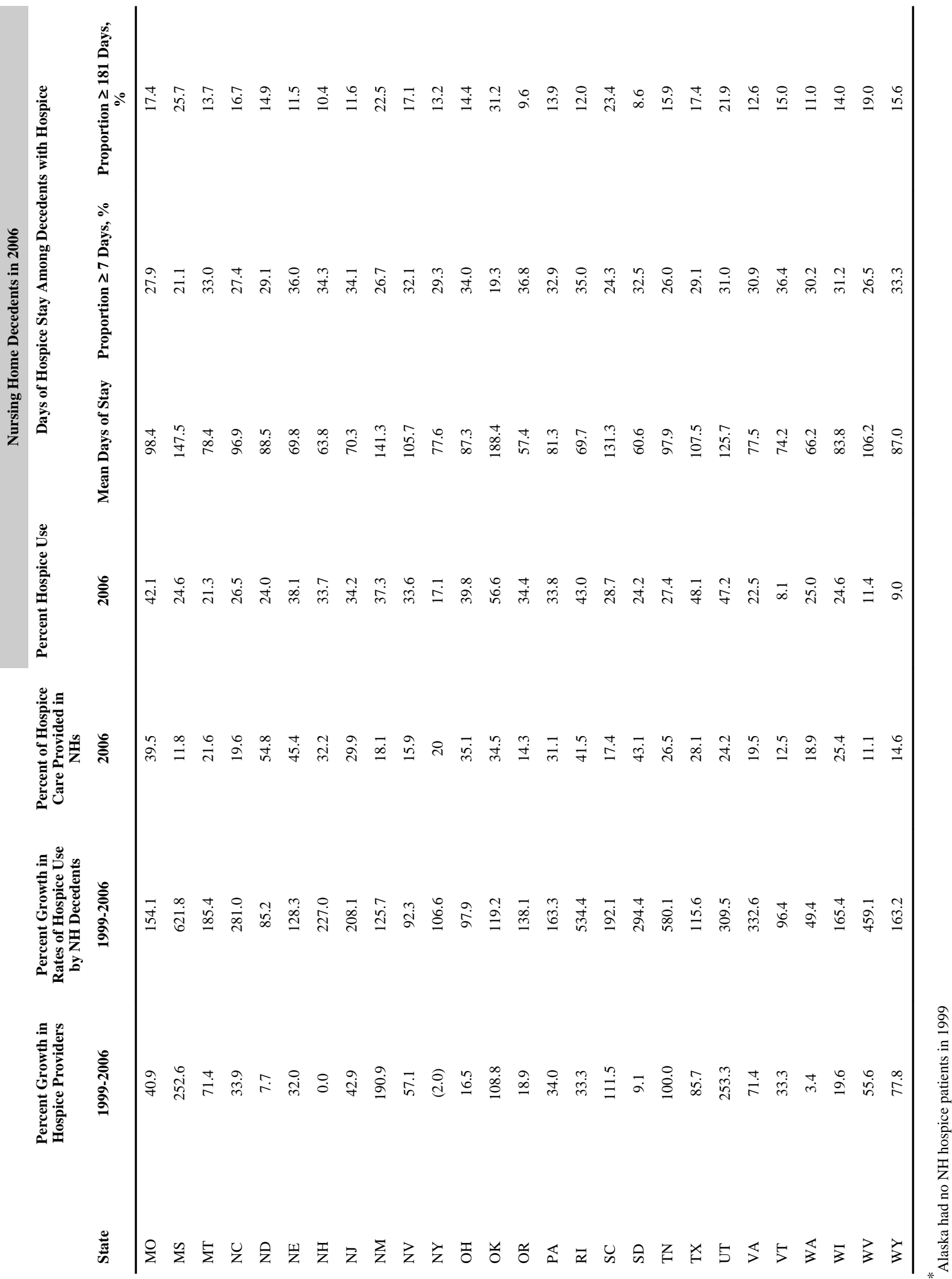

